\title{
Role of ROCK expression in gallbladder smooth muscle contraction
}

\author{
BIN WANG, YOU-MING DING, CHUN-TAO WANG and WEI-XING WANG \\ Department of Hepatobiliary and Laparoscopic Surgery, Renmin Hospital of Wuhan University, \\ Wuhan, Hubei 430060, P.R. China
}

Received June 15, 2014; Accepted April 10, 2015

DOI: $10.3892 / \mathrm{mmr} .2015 .3726$

\begin{abstract}
Cholelithiasis is a common medical condition whose incidence rate is increasing yearly, while its pathogenesis has yet to be elucidated. The present study assessed the expression of Rho-kinase (ROCK) in gallbladder smooth muscles and its effect on the contractile function of gallbladder smooth muscles during gallstone formation. Thirty male guinea pigs were randomly divided into three groups: The control group, the gallstone model group and the fasudil interference group. The fasting volume (FV) and bile capacity of the gallbladder (FB) as well as the total cholesterol (TC) and triglyceride (TG) contents of the gallbladder bile were determined. In addition, the gallbladder was dissected to identify whether any gallstones had formed. Part of the gallbladder tissue specimens were used for immunohistochemical analysis of ROCK expression in gallbladder smooth muscles. The results showed that four guinea pigs in the model group and eight in the fasudil group displayed gallstone formation, while there was no gallstone formation in the control group. The FV and FB were significantly increased in the model and fasudil groups. Similarly, the TC and TG contents of gallbladder bile were increased in these groups. The positive expression rate of ROCK in gallbladder smooth muscles in the model and fasudil groups was significantly reduced compared with that in the control group $(\mathrm{P}<0.05)$. The results of the present study indicated that the reduction of ROCK expression in guinea pig gallbladder smooth muscles weakened gallbladder contraction and thereby promoted gallstone formation.
\end{abstract}

\section{Introduction}

During the past decade, a large number of studies have shown that Rho-kinase (ROCK) is one of the major kinases involved in cell movement. It participates in the RhoA-ROCK signaling

Correspondence to: Professor You-Ming Ding, Department of Hepatobiliary and Laparoscopic Surgery, Renmin Hospital of Wuhan University, 238 Jiefang Road, Wuhan, Hubei 430060, P.R. China E-mail: dingym62@163.com

Key words: gallstone, smooth muscle, Rho kinase, fasudil, contractile function pathway and regulates smooth muscle cell contraction by regulating the $\mathrm{Ca}^{2+}$ sensitization mechanism (1-4).

The levels of myosin light chain (MLC) phosphorylation, which is dually regulated by $\mathrm{Ca}^{2+} /$ calmodulin $(\mathrm{CaM})$-dependent myosin light chain kinase (MLCK) and $\mathrm{Ca}^{2+}$-independent myosin light chain phosphatase (MLCP), is an important factor affecting the extent of smooth muscle contraction (5). The regulation of MLC phosphorylation by MLCK causes smooth muscle contraction, whereas the inhibition of MLCP can enhance the extent of MLC phosphorylation and smooth muscle contraction and increase $\mathrm{Ca}^{2+}$ sensitivity, a phenomenon known as $\mathrm{Ca}^{2+}$ sensitization $(6,7)$.

In the RhoA-ROCK signaling pathway, several agonists activate RhoA through a series of mechanisms, leading to ROCK activation and the regulation of $\mathrm{Ca}^{2+}$ sensitization, which enhance gallbladder smooth muscle contraction $(8,9)$. Studies have shown that ROCK inhibitors, including Y-27632 and fasudil, can inhibit smooth muscle contraction $(10,11)$, which prompted us to study the mechanisms by which they affect the RhoA-ROCK pathway and $\mathrm{Ca}^{2+}$ sensitization.

In the present study, changes in ROCK expression in gallbladder smooth muscle cells were assessed and the role of ROCK protein in the regulation of gallbladder contractility during the lithogenic process was investigated using a guinea pig model of gallstone formation. The findings of the present study provided experimental evidence on animals, which contributes to the elucidation of the etiology of gallstone formation and may aid in its prevention.

\section{Materials and methods}

Experimental animals. 30 Male guinea pigs weighing 250-300 g were obtained from the Experimental Animal Center of Renmin Hospital of Wuhan University (Wuhan, China). The animals were randomly divided into the control group, the gallstone model group and the fasudil interference group.

Animal models. Gallstone development was induced in the guinea pigs in accordance with previously described methods(12). The control group was fed a normal diet, whereas the model group and fasudil group were fed a $2 \%$ cholesterol-enriched diet for 60 days. From the 20th day onward, the fasudil group were treated with the known ROCK inhibitor, fasudil (5 mg/kg; Chase Sun Pharmaceutical Co., Ltd., Tianjin, China) 
,by intraperitoneal injection $(0.2 \mathrm{ml}$ fasudil per $100 \mathrm{~g}$ body weight diluted in normal saline) twice a day. Animals in the control group and model group were injected intraperitoneally with the same volume of sterile saline twice daily.

\section{Specimen collection}

Fasting gallbladder volume $(F V)$ and fasting gallbladder bile volume $(F B)$. Five guinea pigs from each group were randomly selected on days 30 and 60 , respectively. After a 12-h fast, the guinea-pigs were anesthetized by intraperitoneal injection of $6 \%$ chloral hydrate $(350 \mathrm{mg} / \mathrm{kg}$; Hubei Hechang Chemical Co., Ltd., Wuhan, China). The longest length and two perpendicular diameters [width $(\mathrm{W})$, height $(\mathrm{H})$ ] of the gallbladder were measured under natural conditions. The cystic duct was blocked with a vascular clamp and the volume of bile drawn from the gallbladder was recorded (FB). The gallbladder bile was stored for determination of total cholesterol (TC) and triglyceride (TG) contents.

Collection of gallbladder wall tissue. After removing the gallbladder, the gallbladder lumen was cut longitudinally to determine whether it contained biliary sludge or gallstones. The gallbladder was washed using cold saline, and the residual tissue in the gallbladder wall was stripped off. A number of gallbladder tissue samples were clipped from the gallbladder body, rinsed three times with phosphate-buffered saline (Wuhan Boster Biological Engineering Co., Ltd., Wuhan, China), fixed with 10\% formaldehyde solution (Hubei Ju Sheng Technology Co., Ltd., Hubei, China), dehydrated and embedded in paraffin (Wuhan Boster Biological Engineering Co., Ltd.) for immunohistochemical analysis. Further tissue samples were snap-frozen in liquid nitrogen and stored at $-80^{\circ} \mathrm{C}$.

\section{Detection of methods}

$F V$ and $F B$. The longest length and two perpendicular diameters $(\mathrm{W}, \mathrm{H})$ of the guinea-pig gallbladder were measured under natural conditions. These results were used to calculate FV using Dodd's ellipsoid formula, V=0.52xLxWxH (ml). A vascular clamp was used to block the cystic duct, and the total volume of the bile drawn from the gallbladders was recorded $(\mathrm{FB} ; \mathrm{ml})$.

$T C$ and $T G$. TC and TG contents were determined using a TC and TG kit (Sigma-Aldrich, St. Louis, MO, USA) on an Bayer Advia 2400 automatic biochemical analyzer; Siement, Germany) using the oxidase and enzyme colorimetric methods, respectively (performed by the Department of Laboratory, Renmin Hospital of Wuhan University, Wuhan, China).

ROCK expression. ROCK expression in the gallbladder smooth muscle tissues was detected using immunohistochemical methods. Immunohistochemistry was conducted according to the manufacturer's instructions of the SP-9000 kit (Boster Biological Technology Co, Ltd., Wuhan, China). The evaluation standard was as follows: All cells were counted in 10 randomly selected high-power fields and semi-quantitative results were evaluated on the basis of the degree of staining and the percentage of stained cells. The paraffin sections were de-waxed using xylene (Shanghai Senfi Chemical Co., Ltd., Shanghai, China) for antigen retrieval by heat-induced retrieval using citrate buffer (Shanghai Gunsuo Biological Technology Co., Ltd., Shanghai, China) and following elimination of the endogenous peroxidase activity, the sections were blocked

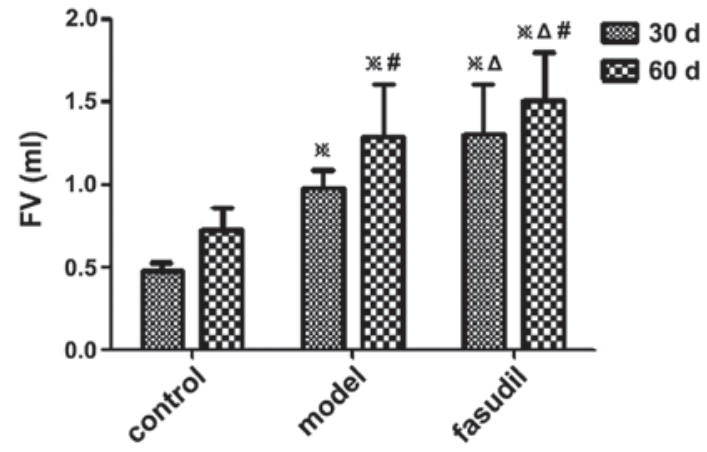

Figure 1. Changes of FV in guinea pigs. FV fasting gallbladder volume.

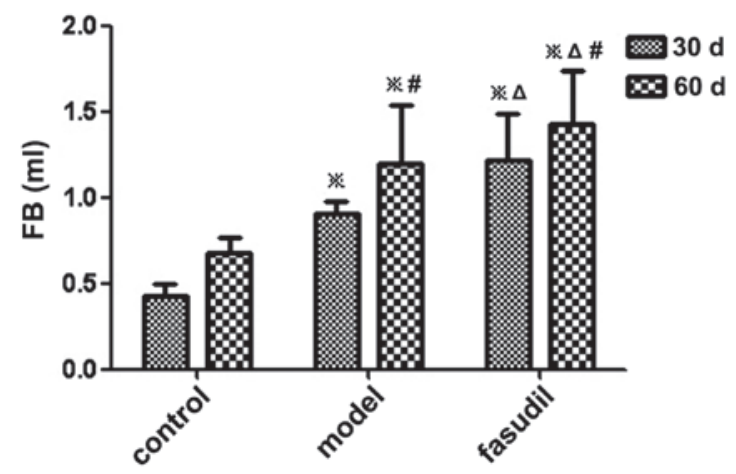

Figure 2. Changes of FB in guinea pigs. FB, volume of bile following fasting.

with goat serum. Next, the unlabeled rabbit anti-mouse polyclonal antibody (cat. no. BA1701-1; Wuhan Boster Biological Engineering Co., Ltd.) primary antibody was added and incubated for 1-2 $\mathrm{h}$. The biotin-labeled goat anti-rabbit polyclonal secondary antibody (cat. no. BA1003; Wuhan Boster Biological Engineering Co., Ltd.) was then added and incubated at $37^{\circ} \mathrm{C}$ for $15 \mathrm{~min}$, followed by horseradish peroxidase-labeled streptavidin for $15 \mathrm{~min}$. The diaminobenzidine chromogenic kit was used to stain the tissue sections, which was followed by re-staining with hematoxylin. The sections were observed under a light microscope (Olympus CX31-LV320; Olympus Co., Ltd., Beijing, China), with the positive cells displaying brown-yellow staining. The degree of cell staining was scored as follows: 0 , no or negligible staining; 1 , pale yellow staining; 2 , brown-yellow staining; 3 , brown staining.

All immunohistochemical tissue sections were analyzed by a computer image analysis program under identical conditions, and the average optical density in the image was regarded as the positive rate of ROCK expression (13).

Statistical analysis. Data were analyzed by SPSS 16.0 statistical and processing software (SPSS, Inc, Chicago, IL, USA). Values are expressed as the mean \pm standard error and were analyzed by one-way analysis of variance, whereas the counts are expressed as percentages and were analyzed using the $\chi^{2}$ test. $\mathrm{P}<0.05$ was considered to indicate a statistically significant difference.

\section{Results}

Changes of the $F V$ and $F B$ and gallstone formation. In the model group, biliary sludge and gallstone formation was 
Table I. Comparison of gallstone formation rate, FV and FB.

\begin{tabular}{|c|c|c|c|c|c|c|c|}
\hline \multirow[b]{2}{*}{ Group } & \multirow[b]{2}{*}{$\mathrm{n}$} & \multicolumn{2}{|c|}{$\begin{array}{l}\text { Rate of gallstone } \\
\text { formation }(\%)\end{array}$} & \multicolumn{2}{|c|}{$\mathrm{FV}\left(\mathrm{cm}^{3}\right)$} & \multicolumn{2}{|c|}{$\mathrm{FB}(\mathrm{ml})$} \\
\hline & & 30 days & 60 days & 30 days & 60 days & 30 days & 60 days \\
\hline Control & 10 & 0 & 0 & $0.48 \pm 0.05$ & $0.73 \pm 0.13$ & $0.43 \pm 0.07$ & $0.68 \pm 0.09$ \\
\hline Model & 10 & $10^{\mathrm{a}}$ & $30^{\mathrm{a}, \mathrm{c}}$ & $0.98 \pm 0.11^{\mathrm{a}}$ & $1.29 \pm 0.32^{\mathrm{a}, \mathrm{c}}$ & $0.91 \pm 0.07^{\mathrm{a}}$ & $1.20 \pm 0.34^{\mathrm{a}, \mathrm{c}}$ \\
\hline Fasudil & 10 & $20^{\mathrm{a}, \mathrm{b}}$ & $60^{\mathrm{a}, \mathrm{b}, \mathrm{c}}$ & $1.31 \pm 0.30^{\mathrm{a}, \mathrm{b}}$ & $1.51 \pm 0.29^{\mathrm{a}, \mathrm{b}, \mathrm{c}}$ & $1.22 \pm 0.27^{\mathrm{a}, \mathrm{b}}$ & $1.43 \pm 0.31^{\mathrm{a}, \mathrm{b}, \mathrm{c}}$ \\
\hline
\end{tabular}

${ }^{\mathrm{a}} \mathrm{P}<0.05$ vs. control group at the same time; ${ }^{\mathrm{b}} \mathrm{P}<0.05 \mathrm{vs}$. the model group at the same time; ${ }^{\mathrm{c}} \mathrm{P}<0.05$ vs. the same group at a different time. $\mathrm{FV}$ fasting gallbladder volume; FB, volume of bile following fasting.

Table II. Comparison of TC and TG in bile.

$\mathrm{TC}(\mathrm{mmol} / \mathrm{l})$

$\mathrm{TG}(\mathrm{mmol} / \mathrm{l})$

\begin{tabular}{|c|c|c|c|c|c|}
\hline \multirow[b]{2}{*}{ Group } & \multirow[b]{2}{*}{$\mathrm{n}$} & & \\
\hline & & 30 days & 60 days & 30 days & 60 days \\
\hline Control & 10 & $0.02 \pm 0.01$ & $0.03 \pm 0.01$ & $0.03 \pm 0.01$ & $0.04 \pm 0.02$ \\
\hline Model & 10 & $0.04 \pm 0.02^{\mathrm{a}}$ & $0.08 \pm 0.02^{\mathrm{a}, \mathrm{c}}$ & $0.07 \pm 0.02^{\mathrm{a}}$ & $0.13 \pm 0.03^{\mathrm{a}, \mathrm{c}}$ \\
\hline Fasudil & 10 & $0.06 \pm 0.02^{\mathrm{a}, \mathrm{b}}$ & $0.11 \pm 0.03^{\mathrm{a}, \mathrm{b}, \mathrm{c}}$ & $0.10 \pm 0.02^{\mathrm{a}, \mathrm{b}}$ & $0.19 \pm 0.04^{\mathrm{a}, \mathrm{b}, \mathrm{c}}$ \\
\hline
\end{tabular}

${ }^{\mathrm{a}} \mathrm{P}<0.05$ vs. control group at the same time; ${ }^{\mathrm{b}} \mathrm{P}<0.05$ vs. the model group at the same time; ${ }^{\mathrm{P}}<0.05$ vs. the same group at a different time. TC, total cholesterol; TG, triglycerides.

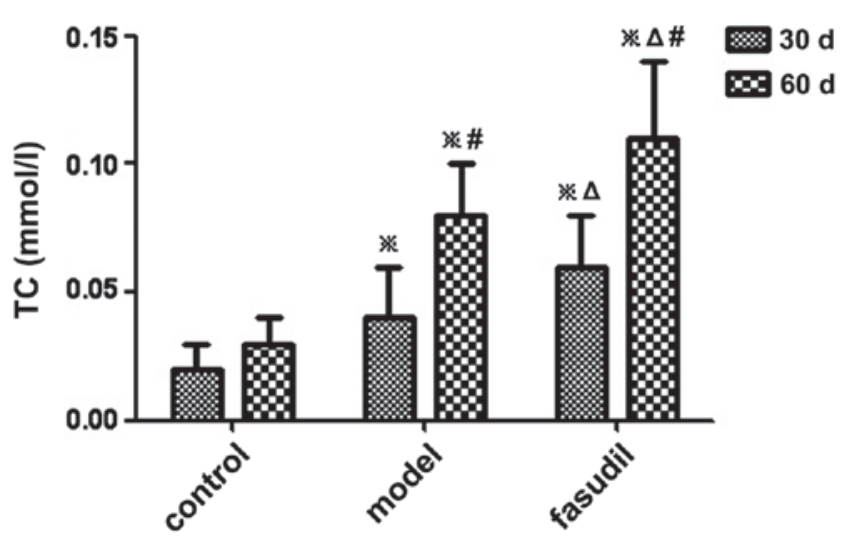

Figure 3. Changes of TC in bile of guinea pigs. TC, total cholesterol.

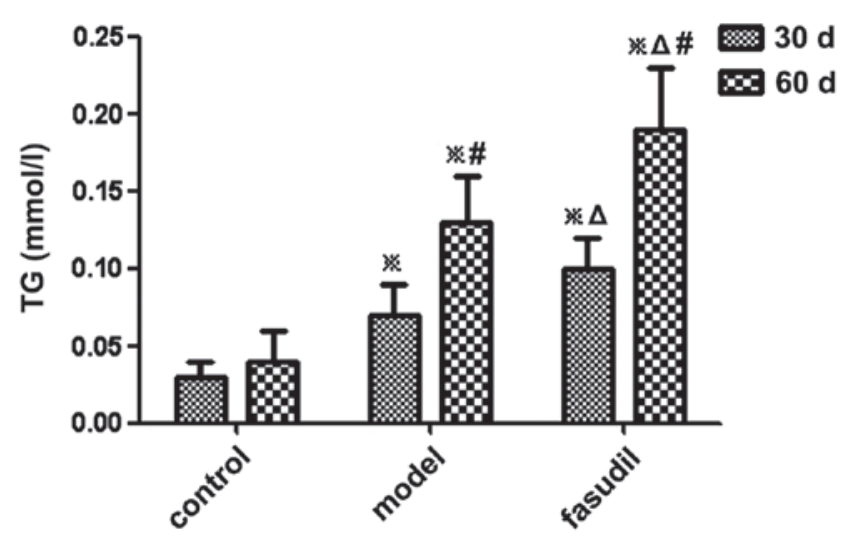

Figure 4. Changes of TG in bile of guinea pigs. TG, triglycerides.

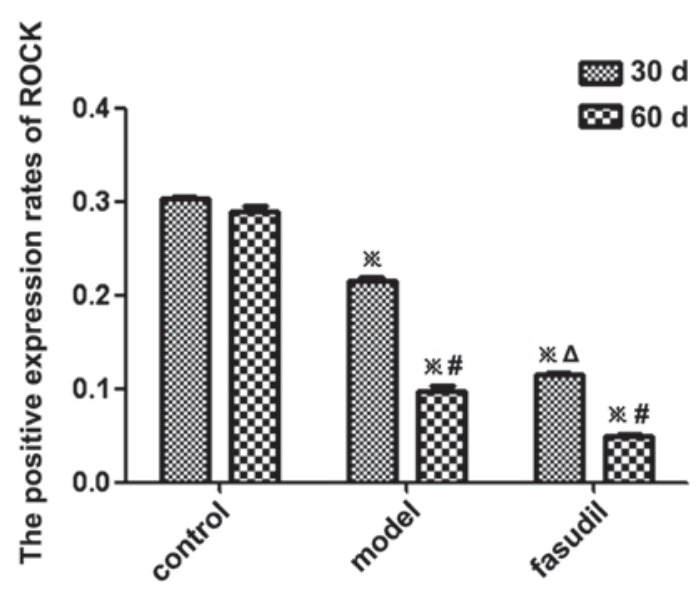

Figure 5. Positive expression rates of ROCK in gallbladder smooth muscles of guinea pigs. ${ }^{*} \mathrm{P}<0.05$ vs. control group at the same time; ${ }^{\Delta} \mathrm{P}<0.05$ vs. the model group at the same time; ${ }^{~} \mathrm{P}<0.05$ vs. the same group at the different time. ROCK-positive expression rates were significantly lower in the model and fasudil group compared with that in the control group. ROCK, Rho-kinase.

observed in one guinea pig on day 30 and in three animals on day 60 , while the two and six counterparts in the fasudil group were affected, respectively.

The FV in the fasudil group was larger than that in the model group at the same time-point. There was a significant difference in the FV and FB between the model group and the fasudil group, and differences between the three groups were also significant $(\mathrm{P}<0.05)$ (Figs. 1 and 2; Table I). 
Table III. Expression of ROCK in gallbladder smooth muscle.

ROCK (OD)

\begin{tabular}{lccc}
\cline { 3 - 4 } Group & $\mathrm{n}$ & \multicolumn{1}{c}{30 days } & \multicolumn{1}{c}{60 days } \\
\hline Control & 10 & $0.3025 \pm 0.0025$ & $0.2889 \pm 0.0056$ \\
Model & 10 & $0.2145 \pm 0.0051^{\mathrm{a}}$ & $0.0981 \pm 0.0046^{\mathrm{a}, \mathrm{c}}$ \\
Fasudil & 10 & $0.1153 \pm 0.0022^{\mathrm{a}, \mathrm{b}}$ & $0.0494 \pm 0.0023^{\mathrm{a}, \mathrm{b}, \mathrm{c}}$
\end{tabular}

${ }^{\mathrm{a}} \mathrm{P}<0.05 \mathrm{vs}$. control group at the same time; ${ }^{\mathrm{b}} \mathrm{P}<0.05 \mathrm{vs}$. the model group at the same time; ${ }^{\mathrm{C}} \mathrm{P}<0.05 \mathrm{vs}$. the same group at a different time. $\mathrm{ROCK}$, Rho-kinase; OD, optical density.

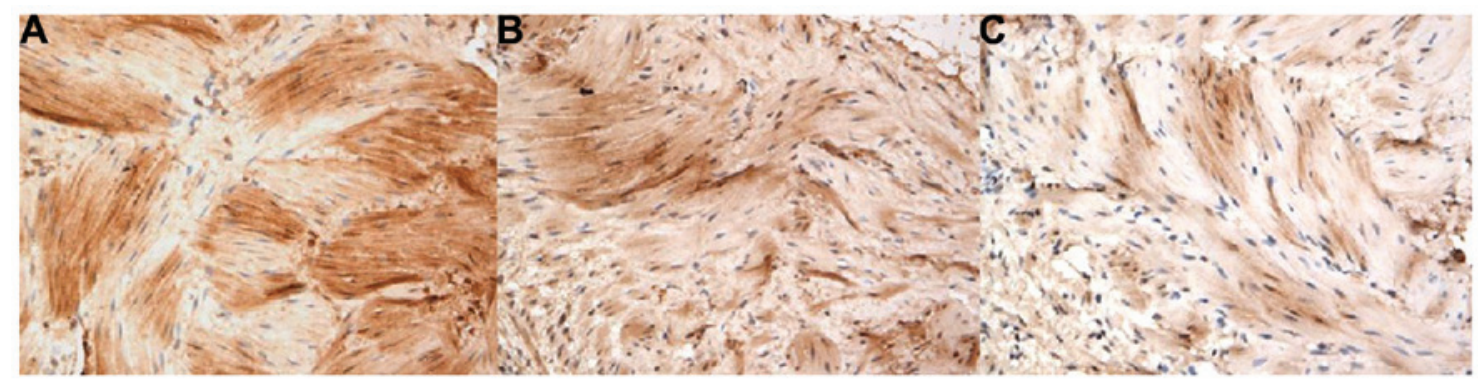

Figure 6. ROCK expression in gallbladder smooth muscles detected by immunohistochemistry. (A) Control group; (B) model group; and (C) fasudil group (magnification, x200). ROCK, Rho-kinase.

Changes in TC and TG contents in gallbladder bile. The levels of TC and TG in the gallbladder bile of guinea pigs were significantly increased in the model group and the fasudil group compared with those in the control group $(\mathrm{P}<0.05)$ (Figs. 3 and 4; Table II).

ROCK expression in gallbladder smooth muscles. The presence of yellow granules indicated positive expression of ROCK in gallbladder smooth muscle cells, and the optical density value, which was obtained using computer image analysis software (Image-Pro Plus 5.0; Media Cybernetics, Inc., Rockville, MD, USA), was used to quantify the expression rate of ROCK. It was observed that the positive expression rate of ROCK in the model group and the fasudil group was significantly lower than that in the control group $(\mathrm{P}<0.05)$ (Figs. 5 and 6; Table III).

\section{Discussion}

Cholelithiasis is a common medical condition worldwide, whose mechanism has not been completely elucidated to date. Its incidence rate is $10-20 \%$ in western countries (2). The incidence of gallstones is increasing along with the improvement of the standard of living conditions and due to rare use of ultrasound and other forms of medical examination, it increased from $2-7 \%$ (3) to $8-10 \%$ (4) today. $>80 \%$ of cholelithiasis cases are gallstone-associated conditions (14). Researchers have attached great importance to the pathological significance of gallbladder in cholesterol-associated gallstone formation in the previous studies (15-17). Studies suggested that gallstone formation was not totally dependent on the physical and chemical changes of bile. The gallbladder, and particularly its dysfunctions regarding its contraction, have a key role in cholesterol-associated gallstone formation (18). In 1983, Doty found that the emptying function of the gallbladder decreased significantly during the formation of cholesterol monohydrate crystals (CMCs) (19). Further evidence provided by flash radiography and ultrasound analysis indicated that the emptying and refilling of the gallbladder with bile and bile metabolism were clearly inhibited in patients with cholecystolithiasis (20).

The present study investigated changes in the expression of ROCK, an important regulatory enzyme in the pathway of gallbladder smooth muscle contraction regulation, and further explored its role in gallbladder smooth muscles contraction function and gallstone formation. The results showed that the FV and FB of guinea pigs were significantly increased after receiving a high-cholesterol diet for up to 60 days. The contents of TC and TG in bile were elevated with increasing time of receiving the high-cholesterol diet. One guinea pig was observed to have gallstones on day 30 , and three animals had gallstones on day 60 , while no gallstones were observed in the control group. This suggested that the increase of the cholesterol concentration in the gallbladder bile may have an important role in gallstone formation. Cholesterol may impair the contraction of the gallbladder smooth muscles, favoring gallstone formation. The same phenomenon was found in fasudil group, in which eight guinea pigs presented with gallstone formation.

Furthermore, ROCK expression in gallbladder smooth muscles was markedly reduced in the model group and the fasudil group. The reduction in ROCK levels may be one of the main reasons for the inhibition of the gallbladder contraction function in guinea pigs following cholesterol intake or fasudil treatment.

The primary mechanism by which ROCK regulates smooth muscle cell contraction is the phosphorylation and de-phosphor- 
ylation of MLC. MLC is phosphorylated by CaM-dependent MLCK and de-phosphorylated by $\mathrm{Ca}^{2+}$-independent MLCP. Therefore, the intracellular elevation of $\mathrm{Ca}^{2+}$ levels results in MLCK activation and subsequent phosphorylation of MLC, finally leading to the contraction of smooth muscle cells $(21,22)$. However, MLC phosphorylation and the tension of smooth muscles do not rely on increased intracellular levels of $\mathrm{Ca}^{2+}$. Certain agonists, including phenylephrine, acetylcholine, U-44619 and endothelin (3-5), can combine with $\mathrm{G}$ protein-coupled receptors, inducing the contraction of smooth muscle cells through enhancing intracellular $\mathrm{Ca}^{2+}$ levels and $\mathrm{Ca}^{2+}$ sensitivity $(23,24)$. The inhibition of MLCP may increase $\mathrm{Ca}^{2+}$ sensitivity in smooth muscle cells and further increase the extent of MLC phosphorylation and smooth muscle tension at a constant $\mathrm{Ca}^{2+}$ concentration, which in turn causes myofilament contraction. When ROCK expression is inhibited or reduced, MLC phosphorylation is decreased, whereas the MLCP activity is enhanced.

In the present study, the expression of ROCK was shown to be reduced alongside gallbladder stone formation following feeding on a high-cholesterol diet or treatment with fasudil. The underlying mechanism is likely to be the inhibitory effect of ROCK on MLCP. The decreased expression of ROCK enhanced the activation of MLCP, decreased the phosphorylation of MLC and weakened the contraction of gallbladder smooth muscle cells. All of the above mechanisms promote cholestasis and gallstone formation. The results of the present study indicated that ROCK expression has an important role in gallbladder contraction function and may be involved in gallstone formation.

In the fasudil treatment group, the expression of ROCK in gallbladder smooth muscles at the same time-point was decreased more markedly than that in the model group, while the rate of gallstone formation was identical in the two groups.

This implied that fasudil is able to significantly inhibit ROCK expression in gallbladder smooth muscles and impair their contraction, therefore inhibiting their emptying function of the gallbladder and favoring gallstone formation as a result. High cholesterol diets as well as fasudil were able to significantly inhibit ROCK expression, leading to the promotion of gallstone formation. Therefore, enhancement of the expression of ROCK in gallbladder smooth muscles may be a novel strategy for the prevention and treatment for cholelithiasis.

\section{Acknowledgements}

The authors would like to thank the Central Laboratory of Renmin Hospital of Wuhan University for excellent technical assistance.

\section{References}

1. Somlyo AP and Somlyo AV: $\mathrm{Ca}^{2+}$ sensitivity of smooth muscle and non-muscle myosin II: Modulated by $\mathrm{G}$ proteins, kinases and myosin phosphatase. Physiol Rev 83: 1325-1358, 2003.

2. Seasholtz TM, Majumdar M and Brown JH: Rho as a mediator of G protein-coupled receptor signaling. Mol Pharmacol 55: 949-956, 1999.
3. Mack CP, Somlyo AV, Hautmann M, Somlyo AP and Owens GK: Smooth muscle differentiation marker gene expression is regulated by RhoA-mediated actin polymerization. J Biol Chem 276: 341-347, 2001.

4. Swärd K, Mita M, Wilson DP, Deng JT, Susnjar M and Walsh MP: The role of RhoA and Rho-associated kinase in vascular smooth muscle contraction. Curr Hypertens Rep 5: 66-72, 2003.

5. Emmert DA, Fee JA, Goeckeler ZM, Grojean JM, Wakatsuki T, Elson EL, Herring BP, Gallagher PJ and Wysolmerski RB: Rho-kinase-mediated $\mathrm{Ca}^{2+}$ independent contraction in rat embryo fibroblasts. Am J Physiol Cell Physiol 286: C8-C21, 2004.

6. Pfitzer G: Regulation of myosin phosphorylation in smooth muscle. J Appl Physiol 91: 497-503, 2001.

7. Camello-Almaraz C, Macias B, Gomez-Pinilla PJ, Alcon S, Martin-Cano FE, Baba A, Matsuda T, Camello PJ and Pozo MJ: Developmental changes in $\mathrm{Ca} 2+$ homeostasis and contractility in gallbladder smooth muscle. Am J Physiol Cell Physiol 296: C783-C791, 2009.

8. Amano M, Ito M, Kimura K, Fukata Y, Chihara K, Nakano T, Matsuura Y and Kaibuchi K: Phosphorylation and activation of myosin by Rho-associated kinase (Rho-kinase). J Biol Chem 271: 20246-20249, 1996.

9. Otto B, Steusloff A, Just I, Aktories K and Pfitzer G: Role of Rho proteins in carbachol-induced contractions in intact and permeabilized guinea-pig intestinal smooth muscle. J Physiol 496: 317-329, 1996.

10. Nobe K and Paul RJ: Distinct pathways of Ca (2+) sensitization in porcine coronary artery: effects of Rho-related kinase and protein kinase $\mathrm{C}$ inhibition on force and intracellular Ca (2+). Circ Res 88: 1283-1290, 2001.

11. Iizuka K, Shimuza Y, Tsukagoshi H, Yoshii A, Harada T, Dobashi K, Murozono T, Nakazawa T and Mori M: Evaluation of Y-27632, a rho-kinase inhibitor, as a bronchodilator in guinea pigs. Eur J Pharmacol 406: 273-279, 2000.

12. Everson GT: Gallbladder function in gallstone disease. Gastroenterol Clin North Am 20: 85-110, 1991.

13. Jian-Ru Yang: Average optical density in quantitative medical image analysis. China JMIT 4: 322-323, 1999.

14. Tzovaras G and Rowlands BJ: Diagnosis and treatment of sphincter of Oddi dysfunction. Br J Surg 85: 588-595, 1998.

15. Portincasa P, Di Ciaula A and vanBerge-Henegouwen GP: Smooth muscle function and dysfunction in gallbladder disease. Curr Gastroenterol Rep 6: 151-162, 2004.

16. van Erpecum KJ, Wang DQ, Moschetta A, Ferri D, Svelto M, Portincasa P, Hendrickx JJ, Schipper M and Calamita G. Gallbladder histopathology during murine gallstone formation: relation to motility and concentrating function. J Lipid Res 47: 32-41, 2006.

17. Lavoie B, Nausch B, Zane E, Leonard M, Balemba O, Bartoo , Wilcox R, Nelson MT, Carey MC and Mawe G: Disruption of gallbladder smoth muscle function is an early feature in the development of cholesterol gallstone disease. Neurogastroenterol Motil 24: 313-329, 2012

18. Carey MC: Pathogenesis of gallstones. Am J Surg 165: 410-419, 1993.

19. Doty JE, Pitt HA, Kuchenbecker SL and DenBesten L: Impaired gallbladder emptying before gallstone formation in the prairie dog. Gastroenterology 85: 168-174, 1983.

20. Jazrawi RP, Pazzip and Petroni ML: Postgrandial gallbladder motor function: refilling and turnover of bile in health and in cholelithiasis. Gastroenterology 109: 582-591, 1995.

21. Sahan-Firat S, Tiftik RN, Nacak M and Büyükafşar K: Rho kinase expression and its central role in ovine gallbladder contractions elicited by a variety of excitatory stimuli. Eur J Pharmacol 528: 169-175, 2005.

22. Quinn T, Feighery R and Baird AW: Role of Rho-kinase in guinea-pig gallbladder smooth muscle contraction. Eur J Pharmacol 534: 210-217, 2006.

23. Uehata M, Ishizaki T, Satoh H, Ono T, Kawahara T, Morishita T, Tamakawa H, Yamagami K, Inui J, Maekawa $M$ and Narumiya S: Calcium sensitization of smooth muscle mediated by a Rho-associated protein kinase in hypertension. Nature 389: 990-994, 1997.

24. Büyükafşar K, Akça T, Nalan Tiftik R, et al: Contribution of Rho-kinase in human gallbladder contractions. Eur $\mathrm{J}$ Pharmacol 540: 162-167, 2006. 